\title{
MANEJO ACTUALIZADO EN EL TRATAMIENTO QUIRÚRGICO DEL CÁNCER DE ESÓFAGO
}

\author{
UPDATED MANAGEMENT SURGICAL TREATMENT OF ESOPHAGEAL CANCER \\ Filomeno Teodoro Jáuregui-Francia', Lissette Jáuregui-Caycho', María del Rosario Figueroa-Bejarano', \\ María del Rosario Jáuregui-Figueroa', Juan Miguel Purilla-Janto
}

\begin{abstract}
RESUMEN
Introducción: El tratamiento quirúrgico del cáncer de esófago sigue siendo un desafío a pesar de los avances en técnica quirúrgica, cuidados pre, post operatorios y complicaciones postoperatorias. Objetivo: Diagnosticar y dar el manejo actualizado en el tratamiento quirúrgico del cáncer de esófago en el hospital nacional Edgardo Rebagliati Martins - EsSalud. Métodos: Se seleccionaron los pacientes con diagnóstico de cáncer de esófago que ingresaron al servicio de cirugía, en el hospital nacional Edgardo Rebagliati Martins - EsSalud, durante el año 2017. Se incluyeron a pacientes de ambos sexos, a partir de 20 años de edad. Para la selección de la muestra se estratificó por edad y sexo de acuerdo al diagnóstico de cáncer de esófago. Resultados: Se diagnosticó 14 pacientes con cáncer de esófago, mediante la historia clínica, exámenes de laboratorio, endoscopia con biopsia, tomografía cervico toraco abdomino pélvica y radiografía esófago estomago duodeno contrastado. No se encontró ningún paciente menor a 40 años, predominó en el sexo masculino, en el grupo etareo entre los 61 y 80 años de edad, todos manifestaron disfagia, odinofagia y pérdida de peso, la complicación post operatoria más frecuente fue fistula de la anastomosis cervical. Conclusión: Se diagnosticaron 14 pacientes con cáncer de esófago, la esofagectomía y la restauración de la continuidad del tracto gastrointestinal son procedimientos complejos y desafiantes. Las fugas anastomóticas y las complicaciones pulmonares son las principales causas de morbimortalidad después de una esofagectomía.
\end{abstract}

Palabras clave: Cáncer de esófago; Cirugía; Tratamiento. (fuente: DeCS BIREME)

\begin{abstract}
Introduction: Surgical treatment of esophageal cancer remains a challenge despite advances in surgical technique, pre-operative care, postoperative care and postoperative complications. Objetive: To diagnose and give updated management in the surgical treatment of esophageal cancer in the national hospital Edgardo Rebagliati Martins - EsSalud. Methods: Patients diagnosed with esophageal cancer who were admitted to the surgery service at the Edgardo Rebagliati Martins - EsSalud national hospital during 2017 were selected. Patients of both sexes were included, from 20 years of age. For the selection of the sample, it was stratified by age and sex according to the diagnosis of esophageal cancer. Results: A total of 14 patients with esophageal cancer were diagnosed by means of the clinical history, laboratory tests, endoscopy with biopsy, cervical thoracic tomography, pelvic abdomino, and contrasted duodenum stomach esophagus. No patient was found less than 40 years old, predominantly in the male sex, in the age group between 61 and 80 years of age, all showed dysphagia, odynophagia and weight loss, the most frequent post-operative complication was fistula of the anastomosis cervical. Conclusion: Fourteen patients with esophageal cancer were diagnosed, esophagectomy and the restoration of the gastrointestinal tract continuity are complex and challenging procedures. Anastomotic leaks and pulmonary complications are the main causes of morbidity and mortality after an esophagectomy.
\end{abstract}

Key words: Esophageal cancer; Surgery; Treatment. (source: MeSH NLM)

'Servicio de Cirugia Oncologica, Hospital Rebagliati, lima-Perú.

Citar como: Filomeno Teodoro Jáuregui-Francia, Lissette Jáuregui-Caycho, María del Rosario Figueroa-Bejarano, María del Rosario Jáuregui-Figueroa, Juan Miguel Purilla-Janto. Manejo actualizado en el tratamiento quirúrgico del cáncer de esófago. [Artículo Original].2018;18(4):59-64. (Octubre 2018). DOI 10.25176/RFMH.v18.n4.1732

(c) Los autores. Este artículo es publicado por la Revista de la Facultad de Medicina Humana, Universidad Ricardo Palma. Este es un artículo de Open Access distribuido bajo los términos de la Licencia Creative Commons Atribución-NoComercial-Compartirlgual 4.0 Internacional.(http://creativecommons.org/licenses/by-nc-sa/4.0/), que permite el uso no comercial, distribución y reproducción en cualquier medio, siempre que la obra original sea debidamente citadas. Para uso comercial, por favor póngase en contacto con revista.medicina@urp.pe 


\section{INTRODUCCIÓN}

El cáncer de esófago es una de las neoplasias más agresivas del tracto digestivo, presenta alta morbilidad y mortalidad. Los tipos histológicos más comunes son el carcinoma de células escamosas (SCC) y el adenocarcinoma $(\mathrm{AC})$, que en conjunto constituyen más del $90 \%$ de las neoplasias esofágicas ${ }^{1}$.

Su incidencia, varía considerablemente, incluso entre las regiones del mismo país. La incidencia más alta se encuentra en China y Sudáfrica (130 por 100.000), siendo el tipo histológico más frecuente el carcinoma de células escamosas; mientras que las cifras más bajas -5 por 100000 habitantes- se registran en Europa y Estados Unidos de Norteamérica, en estos países, el adenocarcinoma del tercio inferior del esófago se ha incrementado significativamente en las últimas tres décadas, llegando a ocupar hasta el $40 \%$ de todas las neoplasias de esófago ${ }^{2}$.

El estadio tumoral es el mejor predictor de supervivencia en los pacientes afectados de neoplasia de esófago. De hecho, el pronóstico de la enfermedad se correlaciona tanto con la presencia de metástasis a distancia, como con el grado de invasión parietal y la afectación ganglionar. Actualmente, la estadificación clínica del cáncer de esófago se realiza mediante el sistema TNM (tumor, nódulo, metástasis), establecido por la American Joint Committee on Cancer 2008)3.

De acuerdo al estadio del cáncer existen diferentes opciones: ¿Cuándo cirugía endoscópica? ¿En qué pacientes? ¿Es útil la neoadyuvancia conociendo los resultados de la experiencia europea con una tasa de respuesta cercana al 50\%? Siendo la cirugía la única alternativa de curación ¿Cuándo cirugía clásica abierta o mininvasiva? son puntos que actualmente están en plena discusión en la literatura mundial.

Nuestro objetivo en el presente trabajo de investigación fue diagnosticar y dar el manejo actualizado en el tratamiento quirúrgico del cáncer de esófago en el hospital nacional Edgardo Rebagliati Martins - EsSalud.

\section{MÉTODO}

\section{Participantes}

De los pacientes que ingresaron al servicio de cirugía, en el hospital nacional Edgardo Rebagliati Martins - EsSalud, fueron seleccionados aquellos con diagnóstico de cáncer de esófago y que firmaron el consentimiento informado para la presente investigación, durante el año 2017. Se incluyeron a pacientes de ambos sexos, femenino y masculino, a partir de 20 años de edad.
Para la selección de la muestra se estratificó por edad y sexo de acuerdo al diagnóstico de cáncer de esófago.

\section{Instrumentos}

Los exámenes pre operatorios que nos permitieron establecer el diagnóstico y tratamiento de cáncer de esófago son: endoscopia alta con biopsia de la lesión, tomografía axial computarizada, radiografía contrastada de esófago estomago duodeno. Los materiales quirúrgicos, equipo de anestesiología y la ropa de sala de operaciones fueron esterilizadas de acuerdo a las normas internacionales vigentes.

\section{Procedimientos}

Los pacientes seleccionados con diagnóstico de cáncer de esófago fueron programados para sala de operaciones de acuerdo a estadio clínico, procediendo con la técnica quirúrgica de acuerdo a la localización del tumor a extirpar.

Para la recolección de datos, se utilizó la historia clínica del paciente, teniendo en consideración: Filiación, enfermedad actual, antecedentes, examen físico y diagnóstico diferencial con otras enfermedades.

Al establecer el diagnóstico y la técnica quirúrgica a realizar, se tuvo especial cuidado en la evolución post operatoria, el tratamiento médico, control, seguimiento y el pronóstico de enfermedad.

Los resultados obtenidos fueron analizados, tabulados y expresados de acuerdo a diagnóstico y tratamiento establecido para cada paciente, teniendo en consideración la edad y el sexo; para expresarlo en tablas y/o gráficos, con la discusión e informe final del trabajo de investigación propuesto.

\section{RESULTADOS}

Los resultados fueron tabulados de acuerdo al diagnóstico y tratamiento establecido para cada paciente, teniendo como referencia la edad y el sexo.

Tabla 2. Sintomatología de cáncer de esófago.

\begin{tabular}{lcc}
\hline SINTOMATOLOGÍA & N $^{\circ}$ DE CASOS & $\%$ \\
\hline Disfagia & 14 & 100.00 \\
\hline Odinofagia & 14 & 100.00 \\
\hline Hiporexia & 10 & 75.00 \\
\hline $\begin{array}{l}\text { Pérdida de peso } \\
\text { Los catorce pacientes presentaron disfagia, odinofagia } \\
\text { y pérdida de peso. }\end{array}$ & 14 & 100.00 \\
\hline
\end{tabular}


Tabla 1. Pacientes con cáncer de esófago.

\begin{tabular}{|cccccc|}
\hline EDAD (AÑOS) & MASCULINO & $\%$ & FEMENINO & $\%$ & $\%$ \\
\hline $41-50$ & 2 & 14.28 & 1 & 7.14 & 21.42 \\
\hline $51-60$ & 2 & 14.28 & 0 & 0.00 & 14.28 \\
$61-70$ & 4 & 28.57 & 0 & 0.00 & 28.57 \\
\hline $71-80$ & 4 & 28.57 & 1 & 7.14 & 35.71 \\
\hline Total & 12 & 85.71 & 2 & 14.28 & 100.0 \\
\hline
\end{tabular}

No se encontró ningún paciente menor a 40 años

correspondió al sexo masculino y el grupo etareo con con cáncer de esófago. Según el sexo el $85.71 \%$ mayor porcentaje fue entre los 61 y 80 años de edad.

Tabla 3. Métodos de diagnóstico en cáncer de esófago.

\begin{tabular}{lcc|}
\hline \multicolumn{1}{|c}{ MÉTODOS DE DIAGNOSTICO } & CASOS & $\%$ \\
\hline Historia clínica y exámenes de laboratorio & 14 & 100 \\
\hline Endoscopia con biopsia & 14 & 100 \\
\hline Tomografía cervico toraco abdomino pélvica & 14 & 100 \\
\hline Rayos X esófago estomago duodeno contrastado & 14 & 100 \\
\hline
\end{tabular}

A todos los pacientes se les realizó el diagnóstico con biopsia, tomografía cervico toraco abdomino mediante la historia clínica, exámenes de laboratorio pélvica y radiografía esófago estomago duodeno pre quirúrgico y otros complementarios, endoscopia contrastado.

Tabla 4. Tratamiento quirúrgico del cáncer de esófago.

\begin{tabular}{|c|c|c|}
\hline TRATAMIENTO QUIRÚRGICO DE ACUERDO A LOCALIZACIÓN EN ESTADIO II Y III & $\mathbf{N}^{\circ}$ DE CASOS & $\%$ \\
\hline $\begin{array}{l}\text { Esófago cervical: Esofagectomía transdiafragmática, transmediastínica posterior, } \\
\text { transposición de tubo gástrico, faringogastroanastomosis Termino terminal, } \\
\text { yeyunostomía transitoria y drenaje }\end{array}$ & 0 & 0.00 \\
\hline $\begin{array}{l}\text { Esófago torácico: Toracoscopía, toracotomía, transposición de tubo gástrico, } \\
\text { faringogastroanastomosis termino terminal yeyunostomía transitoria y drenaje. }\end{array}$ & 2 & 14.28 \\
\hline $\begin{array}{l}\text { Esófago abdominal: Esofagectomía transdiafragmática , transmediastínica } \\
\text { posterior, trasposición de tubo gástrico transmediastínico posterior, } \\
\text { faringogastroanastomosis termino terminal, yeyunostomía transitoria y drenaje. }\end{array}$ & 1 & 7.14 \\
\hline $\begin{array}{l}\text { Esófago abdominal: Esofaguectomía toracoscópica y laparoscópica, trasposición } \\
\text { de tubo gástrico trasmediastínico posterior, esofagogastroanastomosis termino } \\
\text { terminal, yeyunostomía transitoria y drenaje. }\end{array}$ & 2 & 14.28 \\
\hline \multicolumn{3}{|l|}{ Esófago abdominal: Clasificación Swiert Stein } \\
\hline $\begin{array}{l}\text { Tipo } 1 \text { y 2: Esofagectomía transdiafragmática transmediastínica posterior, esófago } \\
\text { gastroanastomosis termino terminal, yeyunostomía transitoria y drenaje. }\end{array}$ & 2 & $14-28$ \\
\hline $\begin{array}{l}\text { Tipo 3: Esofagectomía distal, gastrectomía radical , esófago yeyunostomía } \\
\text { termino lateral, yeyuno yeyuno anastomosis termino lateral y drenaje. }\end{array}$ & 2 & 14.28 \\
\hline Total & 9 & 64.28 \\
\hline
\end{tabular}


Tabla 5. Tratamiento quirúrgico paliativo del cáncer de esófago.

\section{TRATAMIENTO QUIRÚRGICO PALIATIVO ESTADIO IV}

$\mathbf{N}^{\circ}$ DE CASOS

$\%$

Transposición de tubo gástrico en reversa (doble tubo gástrico) por mediastino anterior, esofagogastro anastomosis termino terminal yeyunostomia transitoria y drenaje.

Gastrostomía o yeyunostomía definitiva.

21.42

Total

5

35.71

Recibieron tratamiento quirúrgico paliativo 5 pacientes, de los cuales a 3 pacientes se le realizo gastrostomía o yeyunostomía.

Tabla 6. Tratamiento quirúrgico paliativo del cáncer de esófago.

\begin{tabular}{|c|c|c|}
\hline EVOLUCIÓN & $N^{\circ}$ DE CASOS & $\%$ \\
\hline Buena & 11 & 78.57 \\
\hline Mala & 3 & 21.42 \\
\hline Total & 14 & 100.0 \\
\hline
\end{tabular}

La evolución post operatoria fue buena en 11 pacientes, correspondiendo al $78.57 \%$.

Tabla 7. Complicaciones post operatorias del cáncer de esófago.

\begin{tabular}{|lcc|}
\hline \multicolumn{1}{|c}{ COMPLICACIONES } & No DE CASOS & $\%$ \\
\hline Fistula de anastomosis cervical & 3 & 21.42 \\
\hline Neumonía & 2 & 14.28 \\
\hline Infección de herida operatoria & 1 & 7.14 \\
\hline Total & 6 & 37.50 \\
\hline
\end{tabular}

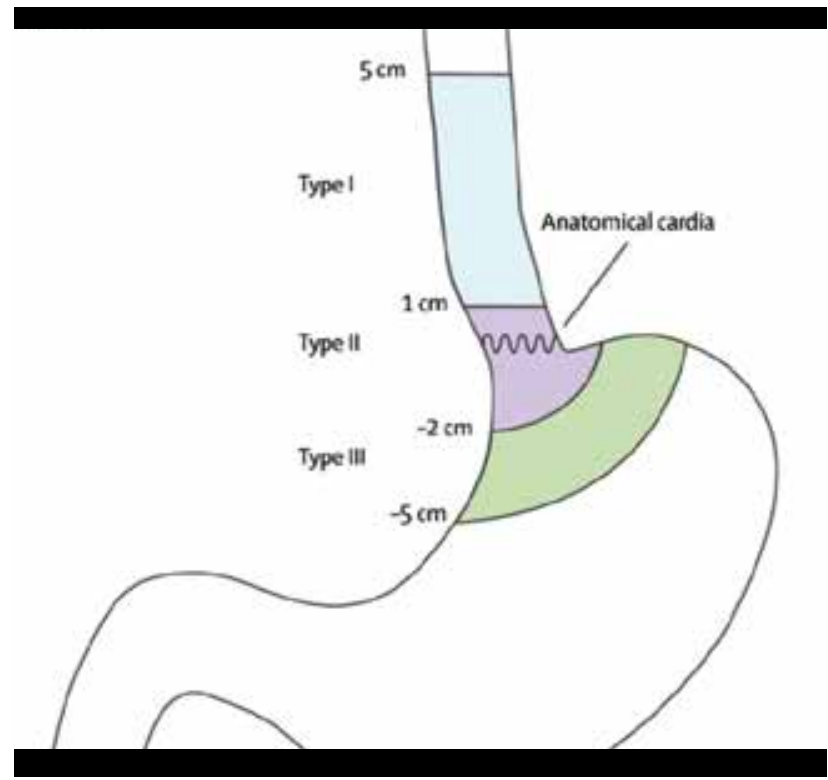

Figura 1. Unión esófago gástrica: Clasificación Siewert - Stein.

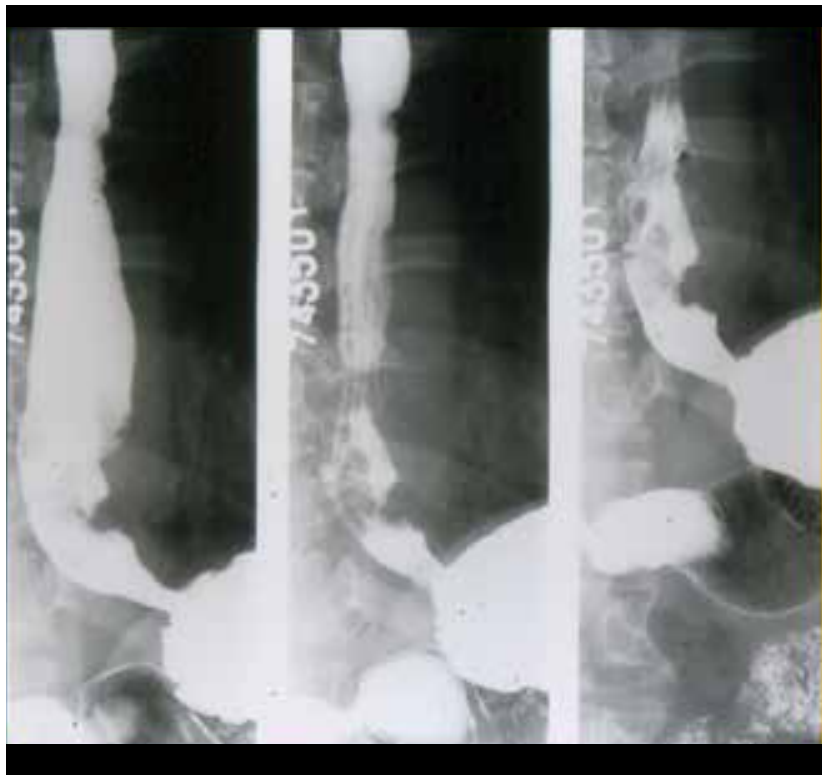

Figura 2. Radiografía esófago estómago contrastada. 


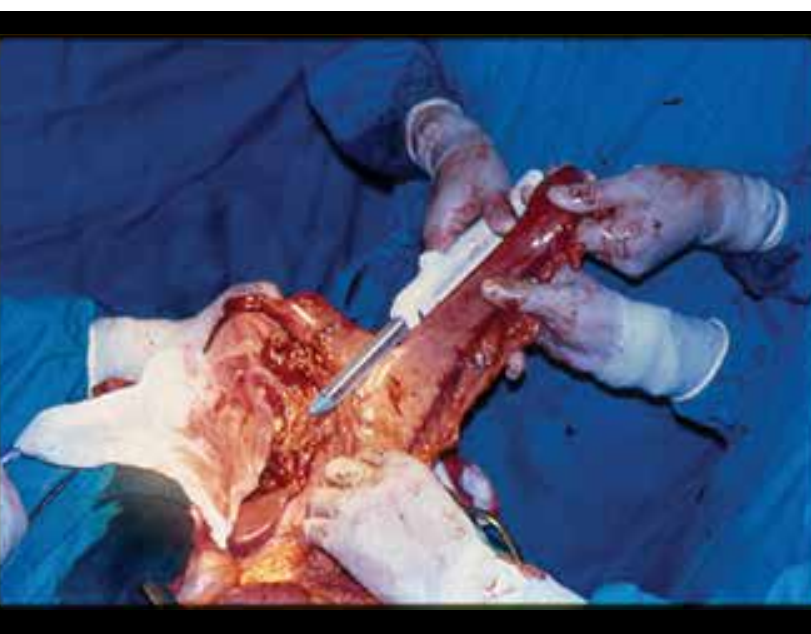

Figura 2. Esofagogastrectomía.

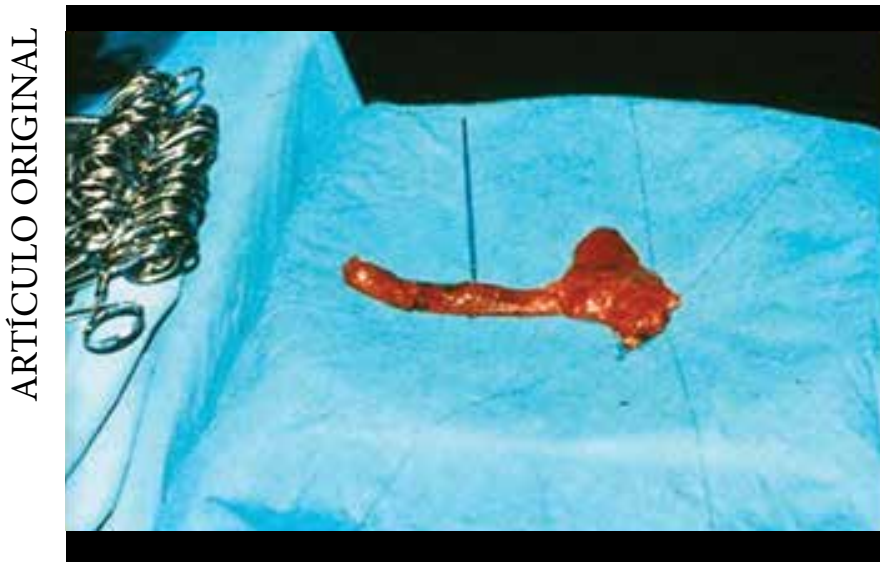

Figura 2. Cáncer de esófago.

\section{DISCUSIÓN}

MMISEl cáncer de esófago es una de las neoplasias asociadas a mayor mortalidad; su mal pronóstico se debe a su diagnóstico tardío ${ }^{4}$. En la actualidad el cáncer de esófago es el tercero más frecuente de las neoplasias gastrointestinales y el séptimo en la lista de los cánceres en el mundo occidental BB, más del $95 \%$ de los casos se deben a dos variedades histológicas: el carcinoma epidermoide o escamoso y el adenocarcinoma.

Es más frecuente en los varones, con una proporción hombre/mujer de 6:1, aunque en poblaciones de alto riesgo la incidencia es igual en ambos sexos 5 .

Los resultados obtenidos de un estudio sobre el tratamiento quirúrgico del cáncer de esófago y cardias durante el período de 1991 a 1995 consistió de 11 pacientes, con mayor predominio entre los 60 y 69 años, el 81,8\% fueron del sexo masculino. Al carcinoma epidermoide correspondieron 6 casos, predominando en el tercio medio, mientras que al adenocarcinoma 5 casos, con localización en el tercio inferior y cardias. La esofagectomía subtotal con sustitución

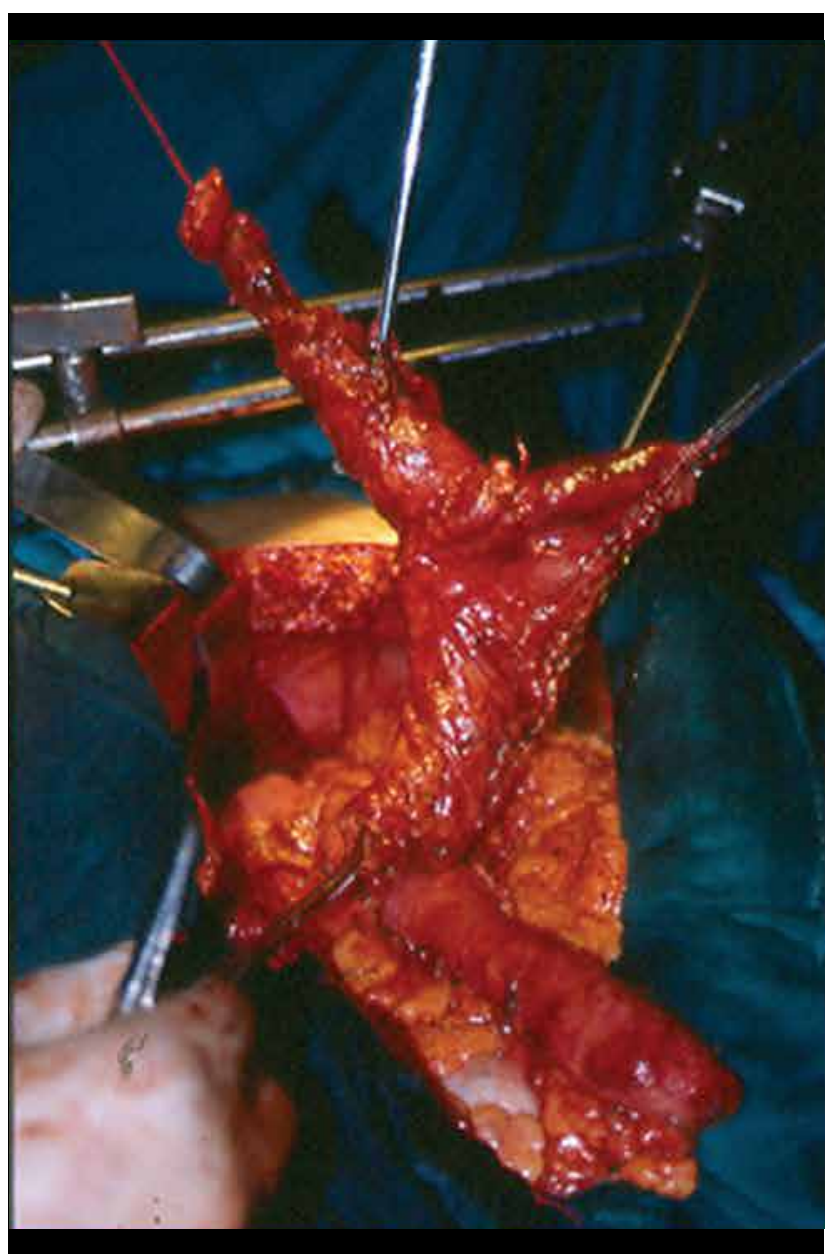

Figura 4. Esofagogastrectomía.

esofágica inmediata fue la técnica más utilizada, y el estómago el órgano empleado mayoritariamente para sustituirlo. El neumotórax intraoperatorio y la sepsis de la herida quirúrgica en las toracotomías fueron las complicaciones más frecuentes, siendo la mortalidad operatoria $36,3 \%$. La supervivencia media de los pacientes que continuaron vivos fue de 13,6 meses $^{6}$.

En otro estudio realizado en el servicio de cirugía del hospital nacional Edgardo Rebagliati Martins - EsSalud; reportaron que de 90 casos correspondientes al manejo quirúrgico del cáncer epidermoide y glandular de esófagoy unión esofagogástrica con infiltración distal del esófago, 51.1\% correspondieron a cáncer epidermoide y $43.3 \%$ a adenocarcinoma; se presentaron dos casos de melanoma primario y uno de linfoma primario, que también fueron sometidos a esofagectomía. Los casos correspondieron a los estadios III y IV, sólo un paciente fue considerado en el estadio I y otro en el estadio II. El $76.5 \%$ de los pacientes tenían antecedentes de tabaquismo, alcoholismo o ambos.

La localización del tumor fue en el 1/3 inferior en el $48.8 \%, 1 / 3$ medio en el $46.6 \%$ y tercio superior en el $4.4 \%$. El $60 \%$ de los pacientes fueron sometidos 
a esofagectomía + gastrectomía del 1/3 superior en block + esofagogastroplastía cervical; el $17.7 \%$ a esofagogastrectomía total + esofagocoloplastía, el $18.8 \%$ a esofagogastroplastía cervical con tubo gástrico confeccionado con curvatura mayor y sin esofagectomía; en un caso se realizó faringotiroidesofaguectomía total con anastomosis a nivel de orofaringe por cáncer epidermoide faringoesofágico, agregando disección radical cervical.

La mortalidad operatoria y perioperatoria fue del $3.3 \%$. La morbilidad fue: respiratoria del $31.1 \%$, fístula cervical de cierre espontáneo del 20\%, mediastinitis severa del 3.3\%. Los pacientes salieron de alta al $15 a v o$ día en promedio; empezando su alimentación oral entre los 10 y 14 días después de la intervención; obteniéndose en todos ellos, un adecuado estado nutricional y mejorando la calidad de vida7. La tasa de supervivencia de los pacientes con cáncer de esófago es precaria. El tratamiento quirúrgico del cáncer de esófago resecable produce tasas de supervivencia a 5 años de 5 a 30\% y tasas de supervivencia más altas en los pacientes de cánceres en estadio temprano. Los tumores pequeños asintomáticos que se limitan a la mucosa o submucosa esofágica se detectan solamente por casualidad. En el caso de estos tumores pequeños, la cirugía es el tratamiento preferido. Una vez que se presentan síntomas (por ejemplo, disfagia, en la mayoría de los casos), los cánceres de esófago habitualmente ya invadieron la capa muscular propia o más allá, y se pueden haber metastatizado a los ganglios linfáticos u otros órganos.

\section{CONCLUSIÓN}

La esofagectomía y la restauración de la continuidad del tracto gastrointestinal son procedimientos complejos y desafiantes. Es imprescindible entender los diferentes procedimientos quirúrgicos y sus potenciales complicaciones post operatorias, siendo las fugas anastomóticas y las complicaciones pulmonares las principales causas de morbimortalidad después de una esofagectomía.
El tipo de resección esofágica se elige en función de la ubicación del tumor, tipo de linfadenectomía, opciones disponibles para la reconstrucción, estado general y función respiratoria del paciente y la experiencia y preferencia del cirujano.

La evolución post operatoria de cáncer de esófago en nuestros pacientes fue buena en el $78.57 \%$.

\section{RECOMENDACIONES}

1. El diagnostico precoz es importante para un tratamiento temprano y oportuno.

2. El diagnostico debe ser confirmatorio con endoscopia alta y biopsia.

3. El tratamiento quirúrgico debe estar determinado de acuerdo al estadio de la enfermedad.

4. Realizar medidas de prevención como endoscopia alta en mayores de 50 años con en personas con factores de riesgo.

5. Determinar y referir a posibles pacientes con este diagnóstico a hospitales de mayor complejidad para confirmación diagnóstica y tratamiento.

6. Realizar seguimiento clínico, con imágenes y endoscopias en los pacientes post operados.

Contribuciones de autoría: Los autores participaron en la generación, recolección de información, redacción y aprobación final del artículo original.

Financiamiento: Autofinanciado.

Conflicto de interés: Los autores declaran no tener conflicto de interés en la publicación de este artículo.

Recibido: 23 de abril del 2018

Aprobado: 05 de octubre del 2018

Correspondencia: Filomeno Teodoro Jáuregui Francia

Dirección: Cal. Santiago Antunez de Mayol Nro. B Int. 71 Urb. Pablo Boner Lima - Lima - La Molina

Celular: 999003894

Correo:filomeno.jauregui@urp.edu.pe

\section{REFERENCIAS BIBLIOGRÁFICAS}

1. Zúñiga Sequeira Tatiana, Cruz Brenes Alejandra. 2012. Revisión bibliográfica de cáncer de esófago .Revista médica de costa rica y Centroamérica LXIX (604) 539-544.

2. Pérez Pereyra Julia, Frisancho Velarde Oscar. 2009 Cáncer de Esófago: Características Epidemiológicas, Clínicas y Patológicas en el Hospital Rebagliati - Lima. Revista de Gastroenterología. Perú 29, (2), 118-123.

3. American Joint Committee on Cancer. 2010. AJCC Cancer Staging Manual. Nueva York: Springer; 2010; 7; 103.

4. Ayala Roberto Nicolás, Graciani Arnaldo Rubén, López Claudio Esteban Morando Félix Rolando. 2007. Cáncer de esófago revisión. Revista de Posgrado de la Vla Cátedra de Medicina. 175 ,17-21.
5. Utrillas Martínez Ana y Del Val Gil José. 2015. Cáncer de esófago Boletín oncológico

6. Valdés Leiva Fidel, Noa Concepcción Rene, Márquez Romero Humberto, Cordero Jiménez Claudio, Estenoz Esquivel Juan Carlos .1996. Tratamiento quirúrgico del cáncer de esófago. Revisión estadística de 5 años. Hospital Provincial Docente de Morón 2 (1).

7. Huamán Guerrero Manuel. 1999. Tratamiento quirúrgico del cáncer de esófago .Revista de la Facultad de Medicina Humana de la Universidad Ricardo Palma. Peru.1 (1). 24-28. 